\title{
Sensitivity analysis of size effect on the performance of hydrostatic bearing
}

\author{
Chen Dongju ${ }^{\text {1a }}$, Dong Lihua ${ }^{1}$, Zhou Shuai $^{1}$ and Fan Jinwei ${ }^{1}$ \\ ${ }^{1}$ College of Mechanical Engineering and Applied Electrics Technology, Beijing University of Technology, Beijing 100124, China
}

\begin{abstract}
For size effect on solid-liquid interface of hydrostatic bearing oil film gap flow in two-dimension, fluid dynamic method is applied to investigate the influence of size effect on bearing capacity, dynamic stiffness and other performances. With the consideration of size effect, Reynolds equation is modified by adopting velocity slip boundary condition into Reynolds equation. The sensitivity factors are used to make a quantitative and qualitative analysis. Numerical simulation results show that size effect will affect bearing performances to a certain degree and the effect curve of size effect on bearing performances are given. The four maximum oil film pressures reduce with the increase of slip length. The maximum sensitivity of bearing capacity is $81.94 \%$.
\end{abstract}

\section{Introduction}

Hydrostatic bearings are widely used in precision machines. Oil film of hydrostatic bearing plays an important role in balancing external load, reducing friction, eliminating wear and so on. With the consideration of the scale division of conventional liquid flow, the research of oil film flow of hydrostatic bearing has entered into micro-scale. However, the performance of liquid flow in micro-scale will be different with the one in macro-scale. Size effect on the one hand behaves of the ratio increase of fluid surface area to volume ratio, when the scale is transferred from macro-scale $(\mathrm{cm} \sim \mathrm{m})$ to micro-scale (um $\sim \mathrm{mm}$ ). Compared to the volume, the ratio of surface area increases from $102 \mathrm{~m}-1$ to $106 \mathrm{~m}-1$, which will accelerate the process of heat transfer on the surface, thereby the temperature rise of the film will be reduced; on the other hand shows a velocity gradient effect, i.e. the shear stress, which along the surface normal is increased since size shrink. At the same time, shear force improves 103 times in micro-scale. If the shear stress in solid-liquid interface exceeds the limit value, the velocity slip occurs inevitably.

Theoretically, oil film in micro-scale is more likely to slip in solid-liquid interface than in macro conditions, and slip is validated by experiments $[1,2]$. Velocity slip will affect the shape and pressure distribution of oil film [3], thereby affecting oil film flow, bearing capacity, bearing dynamic stiffness and other performances parameters of hydrostatic bearing, and more seriously, oil film is prone to collapse and failure [4-6]. Janez Lupse et al [7] extended boundary element method for the discretisation of the incompressible Navier-Stokes equations in the velocity-vorticity form. Paper [8] also points out that because of the size effect of oil film thickness,

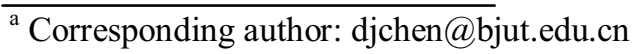

macroscopic tribology law in a way is no longer applicable. In specific friction conditions, material conventional properties cannot be used as the research basis in the analysis of tribology performance, therefore, the influence of size effect on the material properties should be fully considered [9]. At present, the research of the size impact on friction performance and temperature rise of hydrostatic bearings has not been conducted extensively. Thus, it is very necessary to put slip theory to the performance calculation of hydrostatic bearing.

In this paper, the influence of size effect on the performance of hydrostatic bearing is researched. First, the Navier velocity-slip boundary conditions are applied to fluid continuous flow equation, thus the Reynolds equation is modified in micro-scale. Next, according to the principle of oil film flow conservation and modified Reynold, bearing performances are obtained. Then, comparing the result with non-slip model, the influences of size effect on hydrostatic bearing performances are given. This provides a new method for further research on liquid flow in micro-scale.

\section{Analysis of oil film flow characteristics in micro-scale}

\subsection{Model the oil film flow}

Hydrostatic bearing can be divided into two basic categories: recessed hydrostatic bearing and unrecessed hydrostatic bearing. This article puts four pockets recessed liquid radial hydrostatic bearing as the investigated object and its structure is shown in Figure 1. Lubricating oil is pressured into the bearing pocket 
through the restrict orifice (as shown in Figure 2 (a)), until the oil pocket is full, and then discharges from circumferential, axial sealing surface and is returned to channel. The lubricating oil flow in bearing clearance is just like the flow in two plate gap shown in Figure 2 (b), and the coordinate system is established as shown in Fig 2 (b).1, 2 represent journal and bush surface and $\mathrm{V}$ represents line speed of rotating spindle. $\mathrm{X}, \mathrm{Y}$, and $\mathrm{Z}$ respectively represent circumferential, radial and axial direction of bearing oil film.

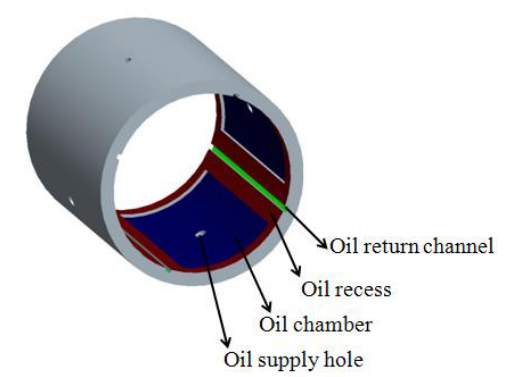

Figure 1 The structure of hydrostatic bearing

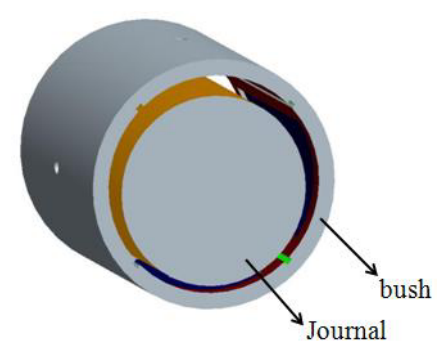

(a)

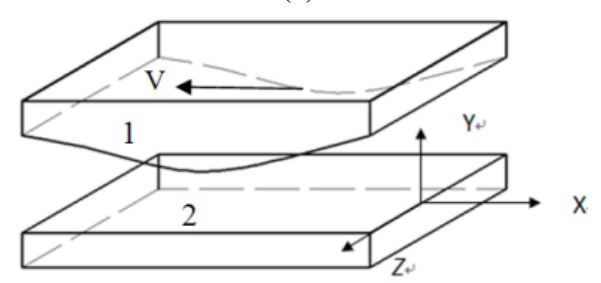

(b)

Figure 2 Oil film flow in hydrostatic bearing clearance and coordinates establishment

Assuming lubricating oil is Newtonian fluid, lies in laminar flow state, and the pressure along film thickness direction remains constant. So the Navier-Stokes equation can be simplified as Eq. (1) and the isoviscous 2-D Reynolds equation is derived from it.

$$
\left\{\begin{array}{l}
\frac{\partial p}{\partial x}=\frac{\partial \tau_{y x}}{\partial y}=\frac{\partial}{\partial y}\left(\eta \frac{\partial u}{\partial y}\right)=\eta \frac{\partial^{2} u}{\partial y^{2}} \\
\frac{\partial p}{\partial y}=0 \\
\frac{\partial p}{\partial z}=\frac{\partial \tau_{y z}}{\partial y}=\frac{\partial}{\partial y}\left(\eta \frac{\partial w}{\partial y}\right)=\eta \frac{\partial^{2} w}{\partial y^{2}}
\end{array}\right.
$$

where $p$ is oil film pressure, $\tau_{y x}$ and $\tau_{y z}$ is oil film shear stress along $\mathrm{X}$ direction and $\mathrm{Y}$ direction, $u$ and $w$ is oil film speed along $\mathrm{X}$ direction and $\mathrm{Z}$ direction, $\eta$ is fluid viscosity.
It requires fixed boundary conditions to solve Eq. (1), namely reflecting the effect of micro-scale velocity slip boundary conditions.

\subsection{Velocity slip boundary condition in two directions}

With the improvement of testing techniques in micronano level, it was found that the size effect of micro-scale makes it easier to the occurrence of velocity slip in the solid-liquid interface.

Currently, a lot of researches on the model of velocity slip have been made, they are: Navier slip model [10], nonlinear slip model [11], ultimate shear force model [4] and so on. Navier slip model is suitable for the occasion of lower shearing rate and the most widely used at present. In this paper, Navier slip model is adopted to analyze oil film flow in hydrostatic bearing.

In Navier slip model, it assumes that fluid velocity is proportional to the shear stress, that is

$$
v_{s}=\left.b \frac{\partial v_{x}}{\partial y}\right|_{\text {wall }}
$$

where $v_{x}$ is fluid velocity along $\mathrm{X}$ direction, $\mathrm{Y}$ is the normal direction of the interface, $b$ is called slip length, which is the distance between actual surface and assumed surface, shown in Figure 3. The size of slip length is determined by some attribute parameters such as material properties, shear rate and gas layer existing in solid-liquid interface, and these factors cannot be simply coupled [12], so slip length is very difficult to determined, generally cannot be obtained by numberical calculation but experiments. Li Xinming used a method of theory combined with practice to study the method of slip length measurement and established a model [13]. According to [14] the slip length in this paper ranges from $0 \sim 7 \mu \mathrm{m}$.

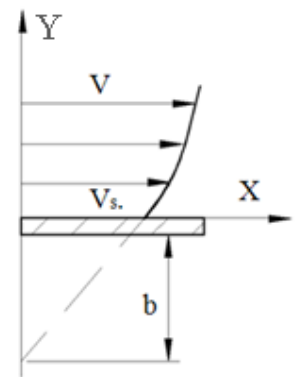

Figure 3 Navier slip mode

According to Navier slip velocity boundary condition, velocity boundary condition of oil flow film on the sealing surface can be written as:

$$
\begin{aligned}
& \text { at } y=0, \quad u=u_{s}=-b_{1 x} \frac{\partial u}{\partial y}, \quad w=w_{s}=-b_{1 z} \frac{\partial w}{\partial y} \\
& \text { at } y=h, \quad u=u_{2}-b_{2 x} \frac{\partial u}{\partial y}, \quad w=w_{s}=-b_{2 z} \frac{\partial w}{\partial y}
\end{aligned}
$$


where $u_{2}$ is line-speed of rotating spindle; $b_{1 x}, b_{1 z}$ are the slip length in $\mathrm{X}$ and $\mathrm{Z}$ directions of bush; $b_{2 x}, b_{2 z}$ are slip length in $\mathrm{X}$ and $\mathrm{Z}$ directions of journal.

\subsection{Modified Reynolds equation by velocity slip boundary condition}

Taken slip boundary condition into consideration, oil film velocity equations in the circumferential and axial direction are gained by the integration of Eq. (1).

$$
\begin{aligned}
& u=\frac{1}{2 \eta} \frac{\partial p}{\partial x} y^{2}-\frac{h^{*} b_{l x}}{\eta} \frac{\partial p}{\partial x}+ \\
& \frac{y}{h}\left(u_{2}-\frac{b_{2 x}\left(h-h^{*}\right)}{\eta} \frac{\partial p}{\partial x}-\frac{h^{2}}{2 \eta} \frac{\partial p}{\partial x}+\frac{h^{*} b_{l x}}{\eta} \frac{\partial p}{\partial x}\right) \\
& w=\frac{1}{2 \eta} \frac{\partial p}{\partial z}\left(y^{2}-y h-h b_{l z}\right)
\end{aligned}
$$

where $h^{*}$ is oil film thickness in axial direction when the shear stress is zero.

The volume of fluid flow through unit width is gained by integrating Eq. (4) along the way of film thickness,

$$
\begin{aligned}
& q_{x}=\int_{0}^{h} u d y= \\
& {\left[-\frac{h^{3}}{12 \eta}-\frac{h b_{2 x}}{2 \eta}\left(h-h^{*}\right)-\frac{h h^{*} b_{l x}}{2 \eta}\right] \frac{\partial p}{\partial x}+\frac{h u_{2}}{2}} \\
& q_{z}=\int_{0}^{h} w d y=\left(-\frac{h^{3}}{12 \eta}-\frac{h^{2} b_{1 z}}{2 \eta}\right) \frac{\partial p}{\partial z}
\end{aligned}
$$

The modified Reynolds is from mass conservation equations [15]:

$$
\frac{\partial}{\partial x}\left(q_{x}\right)+\frac{\partial}{\partial z}\left(q_{z}\right)=-\frac{\partial h}{\partial t}
$$

Assuming that spindle rotated in steady state, modified Reynolds equation is gained by bringing the equation (5) into Eq. (6):

$$
\begin{aligned}
& \frac{\partial}{\partial x}\left\{\left[-\frac{h^{3}}{12 \eta}-\frac{h b_{2 x}}{2 \eta}\left(h-h^{*}\right)-\frac{h h^{*} b_{l x}}{2 \eta}\right] \frac{\partial p}{\partial x}+\frac{h u_{2}}{2}\right\} \\
& +\frac{\partial}{\partial z}\left[\left(-\frac{h^{3}}{12 \eta}-\frac{h^{2} b_{l z}}{2 \eta}\right) \frac{\partial p}{\partial z}\right]=0
\end{aligned}
$$

It can be seen from the modified Reynolds equation that slip in the $\mathrm{Z}$ direction of journal is not introduced into the Eq.(7), that is, the slip in the $\mathrm{Z}$ direction of journal does not affect oil film flow. However, some assumptions must be made about the equation for the further study. Assumptions should be based on current study and specific working conditions, such as material conditions and fluid properties alike. Slip length is assumed to be equal in isotropic material $[15,16]$. This paper assumes $b=b_{1 x}=b_{1 z}=b_{2 x}=b_{2 z}$.
In order to carry out calculations, Eq.(7) should be dimensionless combined with the hypothesis of slip length. Characteristic pressure is $p_{0}$, characteristic slip length and characteristic oil film thickness are bearing radius clearance $c$, characteristic axial length of oil film is half the length of bearing $L / 2$, characteristic axial length of oil film is radius $R$ of bearing. Suppose

$$
\begin{aligned}
& p=p_{0} P \quad, \quad p_{0}=\frac{6 w \eta R^{2}}{h_{0}{ }^{2}}, \quad b=b_{0} c, \quad h=H c, \\
& z=\lambda \frac{L}{2}(-1 \leq \lambda \leq 1), x=R \varphi(0 \leq \varphi \leq 2 \pi), \varphi \text { is position }
\end{aligned}
$$

angle of oil film, the dimensionless Reynolds equation is as equation (8):

$$
\left(1+6 b_{0}\right) \frac{\partial}{\partial \varphi}\left(H^{3} \frac{\partial P}{\partial \varphi}\right)+\left(1+6 b_{0}\right)\left(\frac{2 R}{L}\right)^{2} \frac{\partial}{\partial \lambda}\left(H^{3} \frac{\partial P}{\partial \lambda}\right)=\frac{\partial H}{\partial \varphi}
$$

\section{Calculation of bearing performance}

\subsection{The calculation of oil film flow}

On the calculation of flow of hydrostatic bearing, the existing studies do not take the impact of size effect into account, which have a great difference with actual working conditions. To solve the problem, this paper takes orifice compensated hydrostatic bearing with four pockets as research object, and the main structural parameters is shown in Figure 4. Under the external load the spindle finally stays in the equilibrium position $O_{l}$, the eccentricity of the spindle is $e$, deviation angle is $\theta$, $\theta_{1}, \theta_{2}$ is oil chamber half-width and oil pad half-width, $R$ is radius of spindle, $P_{s}$ is oil supply pressure.

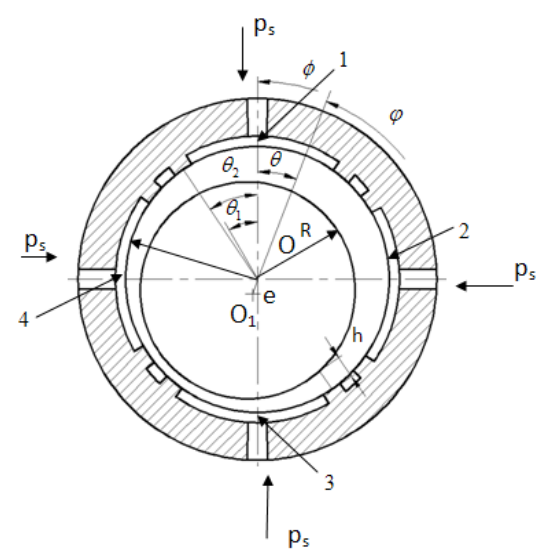

Figure 4 Structural parameters of hydrostatic bearing

When the spindle occurs eccentric, oil film thickness is not constant, and it will change as angular position changes. Oil film thickness equation is given as follow:

$$
h_{l}=h_{0}+e \cos (\varphi)=h_{0}(1+\varepsilon \cos (\phi-\theta))
$$

where $h_{0}$ is the initial film thickness, $\varphi$ is the position angle, $\varepsilon$ is eccentricity ratio, $\varepsilon=e / h_{0}$. 
Combining with the Figure 7, mass flow of each oil pocket can be calculated:

$$
\begin{aligned}
Q_{i a o}= & 2 \int_{\frac{\theta_{1}+\theta_{2}}{2}+\theta}^{\frac{\theta_{1}+\theta_{2}}{2}+\theta} \int_{0}^{h} R w d y d \varphi= \\
& 2 \int_{-\frac{\theta_{1}+\theta_{2}}{2}+\theta}^{\frac{\theta_{1}+\theta_{2}}{2}+\theta} \int_{0}^{h}\left(\frac{1}{2 \eta}\left(y^{2}-y h-b h\right) \frac{\partial p}{\partial z}\right) d y d \varphi \\
Q_{i r o}= & \left.\mathrm{L}\left(-\frac{h_{3}}{12 \eta}-\frac{h^{2} b}{2 \eta}\right) \frac{\partial p}{\partial x}\right|_{h=h_{l}}+ \\
& \left.\mathrm{L}\left(-\frac{h_{3}}{12 \eta}-\frac{h^{2} b}{2 \eta}\right) \frac{\partial p}{\partial x}\right|_{h=h_{r}}+\left.\frac{h u_{2}}{2}\right|_{h=h_{r}}-\left.\frac{h u_{2}}{2}\right|_{h=h_{l}} \\
Q_{i o}= & Q_{\text {iao }}+Q_{i r o}
\end{aligned}
$$

where $Q_{\text {iao }}$ is volume of lubricating oil flow along axial sealing side, $Q_{\text {iro }}$ is the volume of lubricating oil flow along circumferential sealing side, $L$ is the length of bearing, $h_{r}$ is upstream midpoint clearance of oil sealing side of a certain chamber, $h_{l}$ is downstream midpoint clearance of oil sealing side of oil sealing side of a certain chamber.

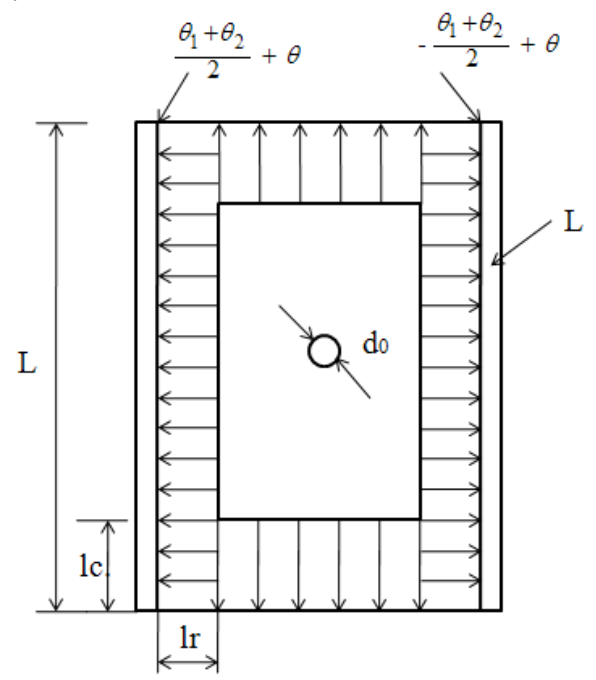

Figure 5 Oil flow out of the hydrostatic bearing chamber

This paper uses orifice restrictor. According to hydrodynamic equations, the flow through orifice restrictor is [17]:

$$
Q_{i i}=\alpha \frac{\pi d_{0}^{2}}{4} \sqrt{\frac{2\left(p_{s}-p_{r i}\right)}{\rho}}
$$

where $Q_{i i}$ is the flow that flows into a certain chamber through orifice restrictor, $\alpha$ is discharge coefficient (0.6 0.7), $\rho$ is the density of lubricating oil, $d_{0}$ is the diameter of orifice restrictor.

Assuming the oil cannot be compressed. The flow flowing into the bearing oil pocket through restrictor is equal to the outflow through sealing surface, thus the flow conservation equation is below:

$$
Q_{i i}=Q_{i o}=Q_{\text {iro }}+Q_{\text {iao }}
$$

\subsection{Calculation of bearing capacity}

Bearing capacity means when oil film formats pressure, the synthetic hydraulic pressure floats the bearing and balances external load acting on the spindle, the synthetic liquid pressure is called load capacity.

The equation of load capacity is as follows:

$$
\begin{aligned}
& W_{x}=\int_{0}^{L} \int_{0}^{2 \pi} R P \cos (\varphi) d \phi d z \\
& W_{y}=\int_{0}^{L} \int_{0}^{2 \pi} R P \sin (\varphi) d \phi d z \\
& W=\sqrt{W_{x}^{2}+W_{y}^{2}}
\end{aligned}
$$

where oil film pressure $P$ is interval function of staging areas, when the integration areas are the chambers $\left(z=[0, l], \phi=\left[-\theta_{1}, \theta_{1}\right],\left[\frac{\pi}{2}-\theta_{1}, \frac{\pi}{2}+\theta_{1}\right]\right.$, $\left.\left[\pi-\theta_{1}, \pi+\theta_{1}\right],\left[\frac{3 \pi}{2}-\theta_{1}, \frac{3 \pi}{2}+\theta_{1}\right]\right)$, the film pressure $P$ is $P_{r i}(i=1,2,3,4)$, When the integration areas are return chutes, the oil film pressure $P$ is zero, when the integration areas are sealing surfaces, the oil film pressure $P$ values as the linear distribution.

According to above analysis, it is easy to determine bearing capacity. This paper provides a convenient method for designing the validation of hydrostatic bearing.

\subsection{Calculation of bearing dynamic stiffness}

Dynamic stiffness of hydrostatic bearing is the ability to resist displacement of the spindle. Bearing oil film stiffness is defined as: when the axis has a tiny displacement, the ratio of the increase of oil film force to the amount of displacement. The displacement and is decomposed into two components $\Delta x$ (along $\mathrm{X}$ direction) and $\Delta y$ (along $\mathrm{Y}$ direction), and the corresponding increase of oil film strength can be decomposed into two components $\Delta W_{x}$ and $\Delta W_{y}$. Four oil film stiffness coefficients are as follows:

$$
\begin{aligned}
& K_{x x}=\frac{\partial W_{x}}{\partial x}, K_{x y}=\frac{\partial W_{x}}{\partial y}, \\
& K_{y x}=\frac{\partial W_{y}}{\partial x}, K_{y y}=\frac{\partial W_{y}}{\partial y}
\end{aligned}
$$

\section{Simulation of oil film performance}

\subsection{Simulation of oil film pressure}

The sensitivity factor of oil film flow $\Delta_{1}$ is defined as following: 


$$
\Delta_{1}=\frac{\left|\left(P_{\max }\right)_{s l i p}-\left(P_{\max }\right)_{n o}\right|}{\left(P_{\max }\right)_{n o}}
$$

where, $\left(P_{\max }\right)_{\text {slip }}$ is the maximum pressure of oil film when the slip occurs, $\left(P_{\max }\right)_{n o}$ is the maximum pressure of oil film when the slip does not happen.

Figure 6 shows the variation of the dimensionless maximum pressure of four pockets $P_{1}, P_{2}, P_{3}, P_{4}$ with the eccentricity in different slip level. It can be seen from Figure 6 that, with the eccentricity increasing, the maximum pressures of the oil pockets $P_{1}, P_{2}, P_{4}$ decrease, and the maximum pressure $P_{3}$ increases. When the eccentricity is constant, the four maximum oil film pressures reduces with the increase of slip length, but the reduction is constantly changing, the sensitivity of maximum oil film pressure can be used to describe the reduced degree of pressure and quantitatively analyzes the influence degree of slip on the maximum pressure. Figure 7 shows the variation of the pressure sensitivities of four pockets $\Delta_{P 1}, \Delta_{P 2}, \Delta_{P 3}, \Delta_{P 4}$ with the eccentricity in different slip level. The maximum pressure sensitivities are $59.31 \%, 21.14 \%, 7.54 \%, 22.31 \%$. Compared Figure 6 with Figure 7, it can be concluded that with the increase of slip length, the four maximum oil pressure sensitivities will increase accordingly.

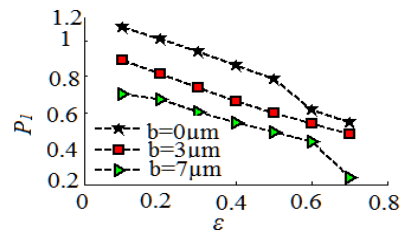

(a) $P_{1}$

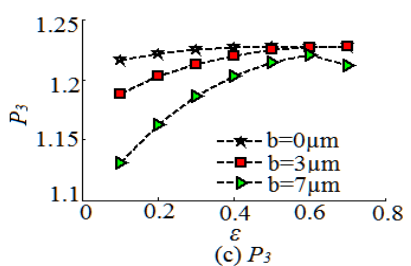

(c) $P_{3}$

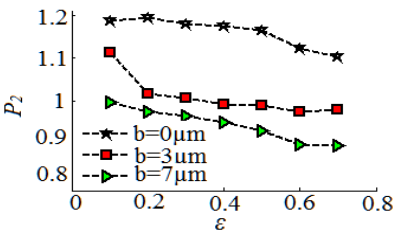

(b) $P_{2}$
Figure 6 Maximum cavity pressure along with eccentricity ratio

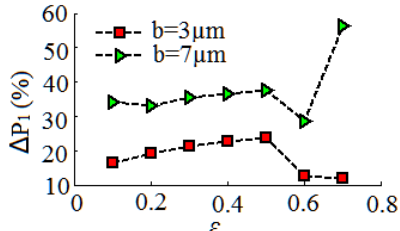

(a) $\Delta \mathrm{P}_{1}$

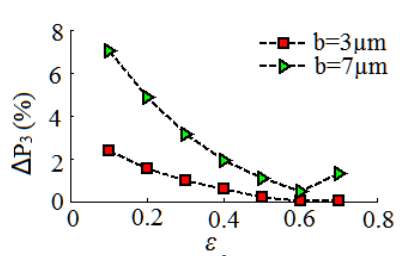

(c) $\stackrel{\varepsilon}{ } \Delta \mathrm{P}_{3}$

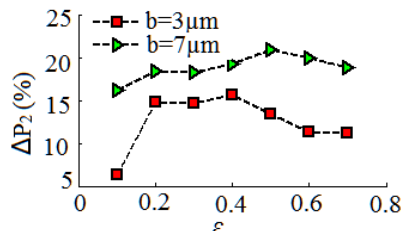

(b) $\Delta \mathrm{P}_{2}$

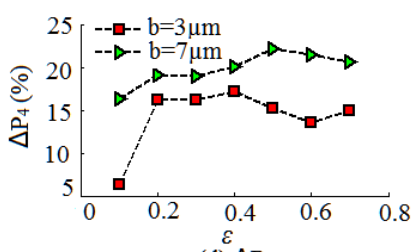

(d) $\Delta \mathrm{P}_{4}$
Figure 7 Maximum cavity pressure sensitivities along with eccentricity ratio

\subsection{Simulation of oil film flow}

$$
\Delta_{2}=\frac{\left|(Q)_{\text {slip }}-(Q)_{n o}\right|}{(Q)_{n o}}
$$

where $(Q)_{\text {slip }}$ is the oil film flow when the slip occurs, $(Q)_{n o}$ is oil film flow when the slip does not happen. $Q_{1}$, $Q_{2}, Q_{3}, Q_{4}$ are the dimensionless oil film flow of four pockets of the bearing. Figure 8 shows the variation of dimensionless oil film flow $Q_{1}, Q_{2}, Q_{3}, Q_{4}$ with the eccentricity of the journal in different slip level. It can be seen from Figure 8 that, with the eccentricity increasing, the flow $Q_{1}, Q_{2}, Q_{4}$ increase, and the flow $Q_{3}$ decreases. When the eccentricity is constant, the four flows increase with the increase of slip length, but the increase rate is constantly changing, the sensitivity factor of flow can be used to describe the increase degree of oil film flow.
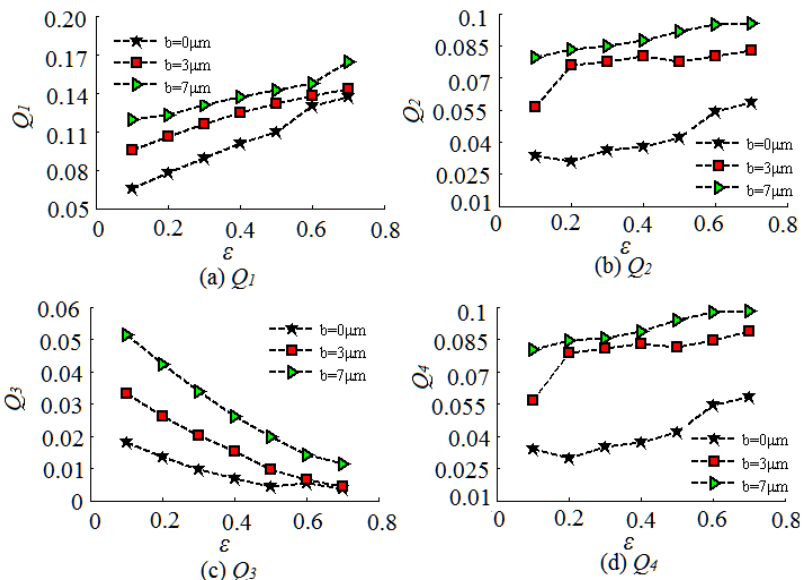

Figure 8 Oil film flow along with eccentricity ratio
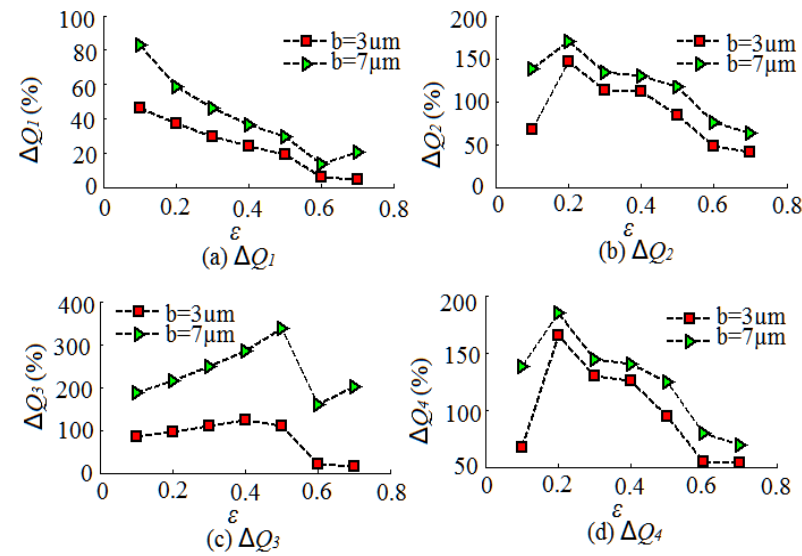

Figure 9 Oil film flow sensitivities along with eccentricity ratio

Figure 9 shows the variation of the oil film flow sensitivities of four pockets $\Delta_{Q 1}, \Delta_{Q 2}, \Delta_{Q 3}, \Delta_{Q 4}$ with the 
eccentricity in different slip level. The maximum flow sensitivities are $84.14 \%, 183.33 \%, 337.24 \%, 183.33 \%$ and the minimum flow sensitivities are $4.67 \%, 40.81 \%$, $16.09 \%, 53.31 \%$. By comparing Figure 8 with Figure 9, it can be concluded that with the increase of slip length, the four oil film flow sensitivities will increase accordingly.

Figure 10 shows the relationship between the total flow $Q$ of the hydrostatic bearing and the rate of eccentricity in different slip case. Under different cases of slip, the total flow increases with increasing eccentricity. The largest relative increase rate of total flow is $57.78 \%$ when the slip does not occur and the slip length is $7 \mathrm{um}$, largest relative increase rate is $22.22 \%$, although the slip does not change the relationship between eccentricity and total flow, the happen of slip weakens the degree of increase of total flow. When the spindle eccentricity is constant, the flow increases with increasing slip length, maximum total flow sensitivity was $100 \%$. This means that the occurrence of slip accelerate flow in bearing clearance, which is conducive to high temperature lubricant rapid outflow of bearings, there is more likely to have a dynamic pressure effect on seal and improve the bearing capacity.

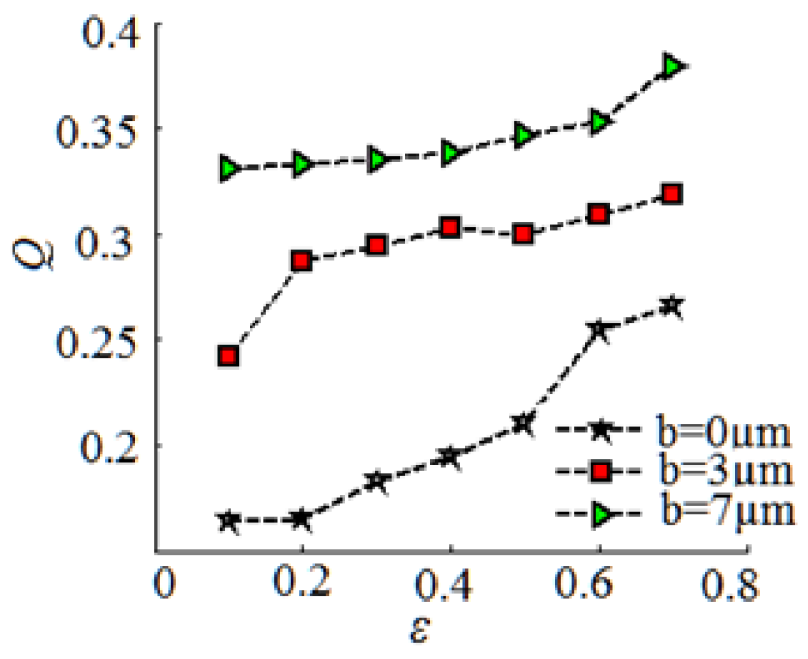

Figure 10 Total flow along with eccentricity ratio

\subsection{Simulation of bearing capacity}

The sensitivity factor of oil film flow $\Delta_{3}$ is defined as following:

$$
\Delta_{3}=\frac{\left|(W)_{\text {slip }}-(W)_{n o}\right|}{(W)_{n o}}
$$

where $(W)_{\text {slip }}$ is bearing capacity when the slip occurs, $(W)_{n o}$ is bearing capacity when the slip does not happen.

Figure 11 shows the relationship between the dimensionless bearing capacity and the eccentricity. It can be seen from Figure 9, when no slip occurs, bearing capacity increases with increase of eccentricity, and the relationship cannot be changed by velocity slip occurred in solid - liquid interface. But when the eccentricity is constant, bearing capacity increases with the increase of slip length, the maximum sensitivity of bearing capacity is $81.94 \%$. Therefore, considering the slip in the design of hydrostatic bearing is different from does not consider, and this shows it is necessary to research the mechanism and influencing factors of slip in oil film clearance flow.

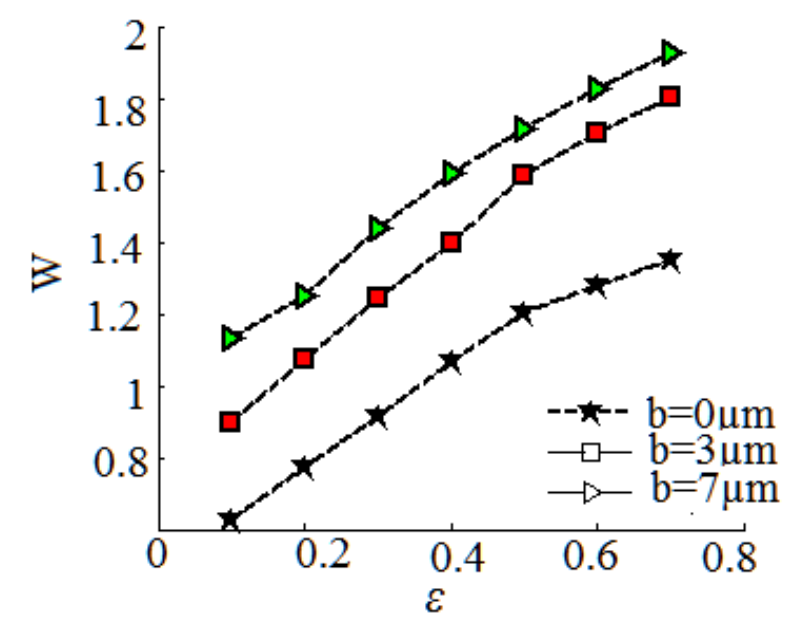

Figure 11 Dimensionless bearing capacity along with eccentricity ratio

\subsection{Simulation of bearing dynamic stiffness}

Dynamic stiffness of hydrostatic bearing is the ability to resist displacement of the flexible shaft moving. In this paper, an incremental method is used to solve the four bearing dynamic stiffness $K_{x x}, K_{x y}, K_{y x}, K_{y y}$. Figure 12 shows the relationship between bearing dynamic stiffness and eccentricity when the slip does not occur. It can be seen from Figure 12, the four bearing dynamic stiffness has the same relationship with eccentricity; with the eccentricity increase, the four dynamic stiffness decreases and proves the cross stiffness $K_{x y}$ and $K_{y x}$ are equal. When the eccentricity is constant, $K_{x x}$ is the largest, followed by the cross stiffness $K_{x y}$ and $K_{y x}, K_{y y}$ is the minimum.

The sensitivity factor of oil film flow $\Delta_{4}$ is defined as following:

$$
\Delta_{4}=\frac{\left|(D)_{s l i p}-(D)_{n o}\right|}{(D)_{n o}}
$$

where $(D)_{\text {slip }}$ is bearing dynamic stiffness when the slip occurs, $(D)_{n o}$ is bearing dynamic stiffness when the slip does not happen.

Figure 13 shows the relationship between four dynamic stiffness $K_{x x}, K_{x y}, K_{y x}, K_{y y}$ and slip length when the eccentricity is 0.3 . Four stiffness increase linearly with slip length decreases, the maximum sensitivities are $3.02 \%, 2.14 \%, 3.30 \%, 3.26 \%$, through quantitative analysis shows that the velocity slip has a certain influence on dynamic stiffness. Therefore, slip should be taken into consideration in the design of 
hydrostatic bearing, especially in the special working conditions the dynamic stiffness is very important.

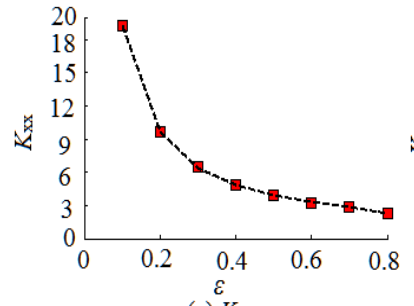

(a) $K_{\mathrm{xx}}$

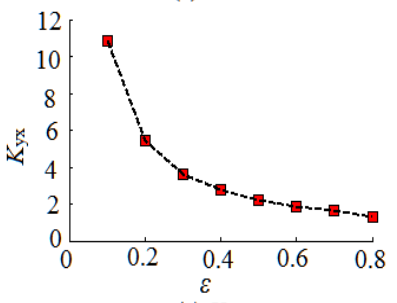

(c) $K_{\mathrm{yx}}$

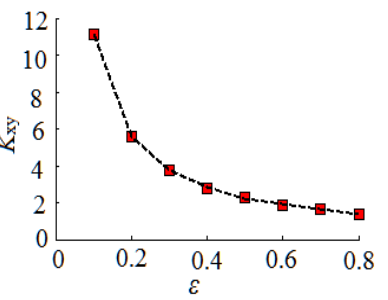

(b) $K_{\text {xy }}$

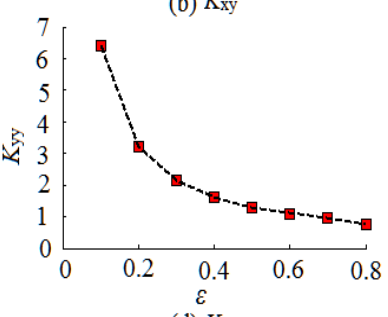

(d) $K_{\text {yy }}$
Figure 12 Dynamic stiffness of no slip along with eccentricity ratio

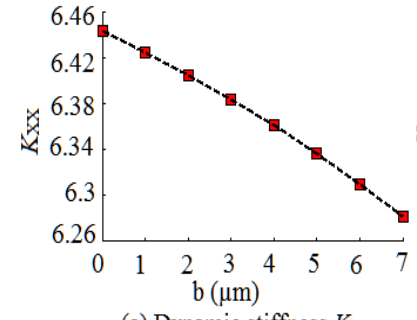

(a) Dynamic stiffness $K \mathrm{xx}$

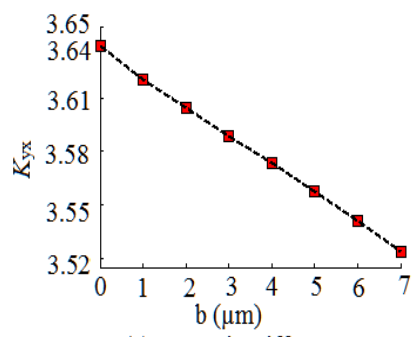

(c) Dynamic stiffness $K_{\mathrm{yx}}$

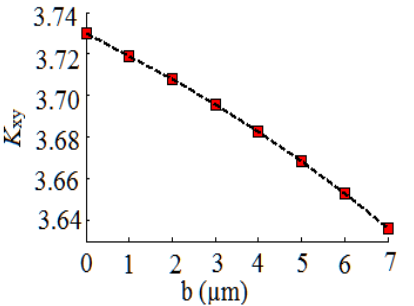

(b) Dynamic stiffness $K_{x y}$

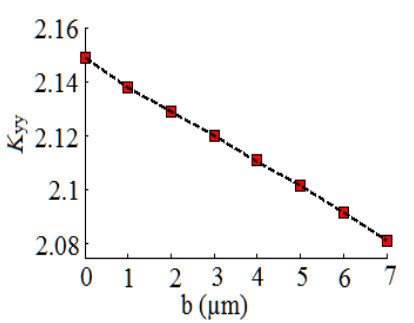

(d) Dynamic stiffness $K_{y y}$
Figure 13 Dynamic stiffness along with slip length for $\varepsilon=0.3$

\section{Conclusions}

This paper analyzes the properties of two-dimensional oil film flow of hydrostatic bearing in micro-scale, and successfully gives flow regularities using Navier slip model. The Reynolds equation is modified by definite boundary conditions in the consideration of velocity slip. Finite difference method is used to solve Reynolds equation, and oil film pressure distribution, bearing capacity, oil film flow, bearing dynamic stiffness are gained. Sensitivity factors of oil film pressure, load capacity, flow and dynamic stiffness are deduced and the variation of them with the eccentricity in different slip length is showed, and the result of the velocity slip reduces bearing dynamic characteristics and stability to some extent is obtained. This proposed the slip should be taken into consideration in the design of hydrostatic bearing, especially in the special working conditions the dynamic stiffness is very important.

\section{Acknowledgements}

This research was funded by the National Natural Science Foundation of China Grant No.51475010, the China Nova program of Beijing and Natural Science Foundation of Beijing Municipality Grant No.3142005.

\section{References}

1. Ol Vinogradova, International Journal of Mineral Processing, 56,31-60 (1999).

2. G Zhang, P Huang, etc., Tribology, 18,243-247 (1998).

3. G Zhang Gu, HR Meng etc., Lubrication. Lubrication Engineering, 4,48-51 (1999).

4. C Wu, Journal of Dalian University of Technology, 33, 172-178 (1993).

5. P Huang,J Luo,S Wen, Acta Mechanica Sinica, 31,745-752 (1999).

6. Z Liu, Journal of Mechanical Engineering,47, 104110(2011).

7. J Lupse, L Skerget, J Ravnik, Journal of Mechanical Engineering, 61,254-264 (2015).

8. Q Xue, J Zhang, Tribology, 14,360-369 (1994).

9. $\mathrm{Z}$ Zhou, Chinese Journal of Mechanical Engineering, 39,22-26 (2003).

10. C Navier, C Navier, Mem.acad.r.sci, 6,389440(1823).

11. P.A Thompson, S.M Troian,etc., Nature, 389,360362(1997).

12. C. Neto, DR Evans., E Bonaccurso, etc., Reports on Progress in Physics, 68,2859-2897 (2005).

13. LI Xin-.Ming, F.Guo, W Pat-Lam, Mocaxue Xuebao/tribology, 32,34-39 (2012).

14. L Ehrenworth, Journal of Fluid Mechanics. 489,5577(2003).

15. CY Chen, QD Chen, WL Li, Tribology International, 61,144-155 (2013).

16. WL Li, HM Chu, MD Chen, Tribology International, 39,1428-1435, (2006). 